\title{
Comparative Karyotypic Studies on Three Species of the Subfamily Viverrinae in Thailand
}

\author{
Alongkoad Tanomtong ${ }^{1}$, Arunrat Chaveerach ${ }^{1, *}$ \\ and Ruengwit Bungongrat ${ }^{2}$

\footnotetext{
${ }^{1}$ Department of Biology, Faculty of Science, Khon Kaen University, Khon Kaen, 4002, Thailand

${ }^{2}$ Genetics Program, Department of Botany, Faculty of Science, Chulalongkorn University, Phayathai, Bangkok 10330, Thailand
}

Received June 1, 2006; accepted June 21, 2006

\begin{abstract}
Summary Comparative karyotypic studies on 3 species, large-spotted civet (Viverra megaspila), large Indian civet ( . zibetha) and small Indian civet (Viverricular indica) using conventional staining and G-banding patterns were investigated. Blood samples taken from Khao Khiew and Dusit Zoo were examined using lymphocyte culture techniques. The results indicate that the number of diploid chromosome of large-spotted civet, large Indian civet and small Indian civet are 38, 38, and 36, respectively. The fundamental number (NF) are 72 for female and 71 for male, 72 for female, and 70 for female and 69 for male. The type number of autosomes, metacentric, submetacentric, acrocentric and telocentric chromosomes are 10-10-12-4, 10-10-12-4 and 10-12-10-2, respectively. Chromosome pairs 15, 17 and 17 are satellite chromosomes. The $\mathrm{X}$ and $\mathrm{Y}$ chromosomes are submetacentric and telocentric for large-spotted civet and small Indian civet, whereas. The $\mathrm{X}$ and $\mathrm{Y}$ chromosome are not known for large Indian civet. In addition, G-banding techniques show that there are 143, 148 and 145 banding patterns which are able to chromosomal classify of these 3 species.
\end{abstract}

Key words Chromosome, G-banding, Karyotype, Large-spotted civet (Viverra megaspila), Large Indian civet (V.zibetha), Small Indian civet (Viverricular indica).

Large-spotted civet, large Indian civet and small Indian civet are members of family Viverridae. The family has only 3 subfamilies, 9 genera and 11 species in Thailand. Really, Thailand is a country with high biodiversity, resulting from its varying ecosystems and forests. It has been estimated that there may be up to 100,000 wildlife species in Thailand as well as many unknown plant species. Unfortunately, rushed economic development and social welfare has led to vast destruction of Thailand's forest and wild life resources. Many wildlife species have become extinct due to loss of habitat, hunting for food, trade and export. One such example is civets, whose numbers are rapidly deteriorating. Masked palm civet, binturong, Asian palm civet and small-toothed palm civet are members of the subfamily Paradoxurinae whereas large-spotted civet, large Indian civet and small Indian civet are members of the subfamily Viverrinae. These civets are in the present rarity. Their situation causes as well great difficulty in obtaining blood samples for cytogenetic studies.

Wuster and Benirschke (1967) studied the cytogenetics of masked palm civet, binturong, and Asian palm civet, and their results showed the number of diploid chromosome 44, 42, and 42, respectively. The karyotypes indicate that masked palm civet have 22 metacentrics and submetacentrics, 20 acrocentrics and telocentrics, and metacentric and submetacentrics $\mathrm{X}$ and Y chromosomes. Binturong shows NF of 68 for both male and female and karyotypes of 22 metacentrics and submetacentric, 18 acrocentrics and telocentrics, and medium metacentric and small metacentric $\mathrm{X}$ and Y chromosomes. Asian palm civet was reported to have NF of 66 for both male and female, $\mathrm{X}$

* Corresponding author, e-mail: raccha@kku.ac.th 


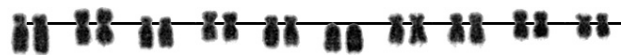
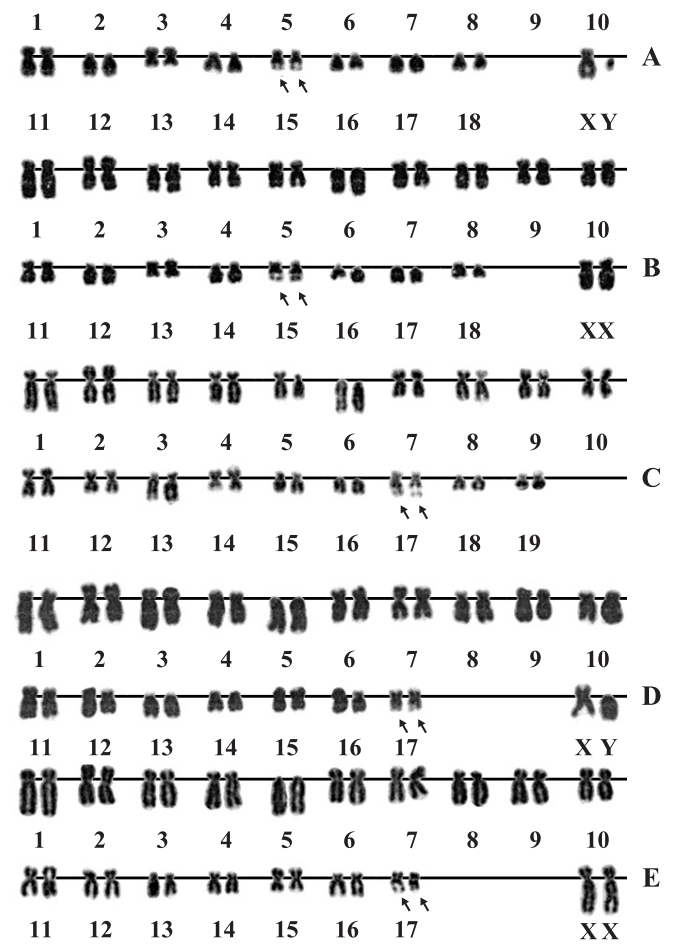

Fig. 1. Karyotype showing different all types of chromosomes for male (A) and female (B) of largedspotted civet (Viverra megaspila) $2 n=38$, female (C) of large Indian civet (Viverra zibetha) $2 n=38$, male (D) and female (E) of small Indian civet (Viverricular indica) $2 n=36$ and satellite chromosomes (arrows) by conventional staining method.
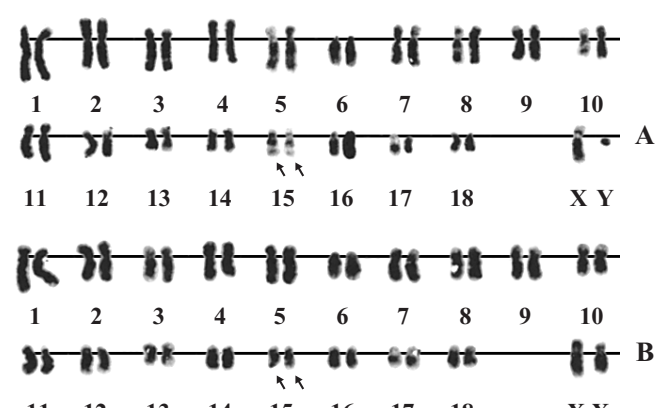

$\begin{array}{lllllllll}11 & 12 & 13 & 14 & 15 & 16 & 17 & 18 & \text { X X }\end{array}$
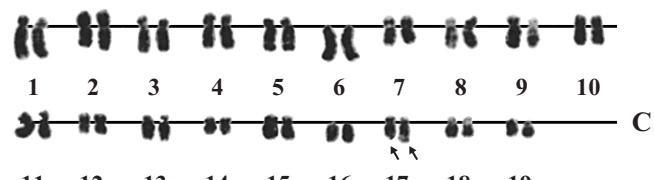

$\begin{array}{lllllllll}11 & 12 & 13 & 14 & 15 & 16 & 17 & 18 & 19\end{array}$
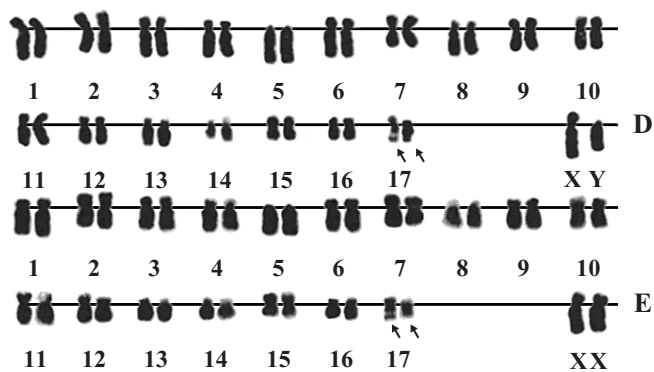

Fig. 2. Karyotype showing different all types of chromosomes for male (A) and female (B) of largedspotted civet (Viverra megaspila) $2 n=38$, female (C) of large Indian civet (Viverra zibetha) $2 n=38$, male (D) and female (E) of small Indian civet (Viverricular indica) $2 n=36$ and satellite chromosomes (arrows) by using G-banding method.

and the Y chromosomes as medium metacentric, and the smallest chromosome was undetermined.

Wang et al. (1984) reported chromosome numbers for masked palm civet, binturong, and Asian palm civet to be $2 n=44,42$, and 42 , respectively. The karyotype of masked palm civet shows 24 metacentrics and submetacentrics, 18 acrocentrics, and the $\mathrm{X}$ and $\mathrm{Y}$ chromosomes are submetacentric and acrocentric. The NF is 69 and 68 for the male and female.

Cytogenetic study on masked palm civet was conducted by Masashi and Harumi (1993). Their results showed chromosome numbers of 44 . The karyotype showed 8 metacentrics and submetacentrics, 18 acrocentrics and 16 telocentrics, and the $\mathrm{X}$ and $\mathrm{Y}$ chromosomes are metacentrics. The NF of both male and female is 66 .

Studies of chromosomal features in Indian muntjacs (Muntiacus muntjak) and Fea's muntjacs (M. feae) of Thailand (Tanomtong et al., 2005a) indicate chromosome variation with breakage, fusion and lacking of homologous chromosome in the same species and between sexes. These factors cause abnormalities in productive gametes and may have contributed to the Fea's muntjacs becoming an endangered species.

The most recent cytogenetics was studied by Tanomtong et al. (2005a, b) on wild animal species of the subfamily Paradoxurinae in Thailand including masked palm civet, binturong, Asian palm civet and small-toothed palm civet, and their results showed the number of diploid chromo- 


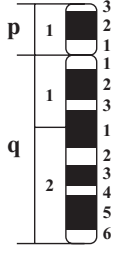

1
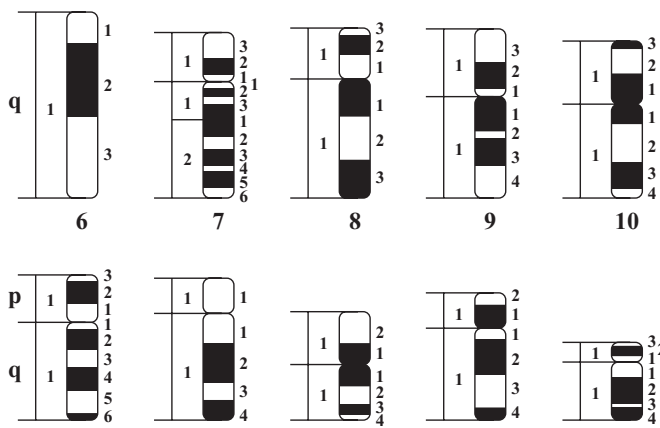

12

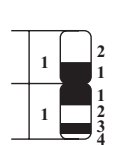

13

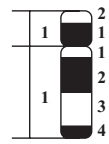

14

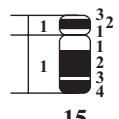

15

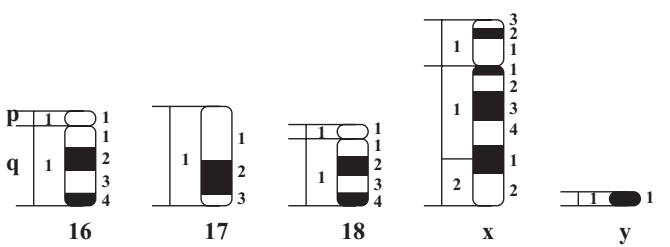

Fig. 3. Idiograms showing lengths and shapes of chromosomes for large-spotted civet (Viverra megaspila) $2 n=38$.
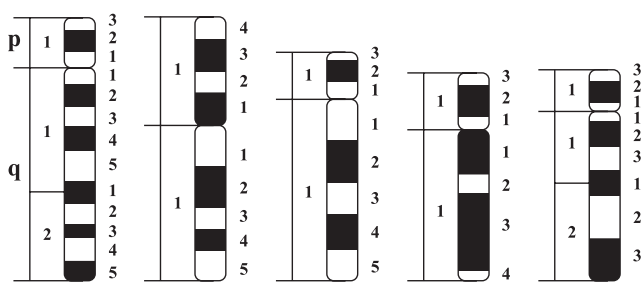

4

5
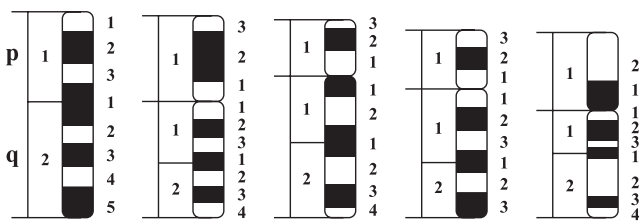

8

10
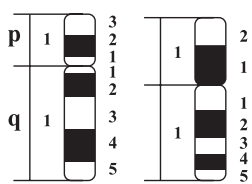

12

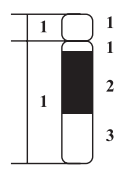

13

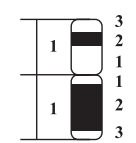

14

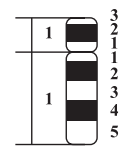

15

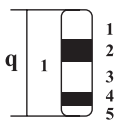

16

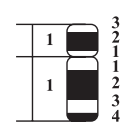

17

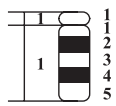

18

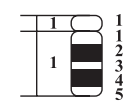

19

Fig. 4. Idiograms showing lengths and shapes of chromosomes for large Indian civet (Viverra zibetha) $2 n=38$.

some 44, 42, 42 and 40, respectively. The NF is 62,61 and 50 for both male and female. The type number of autosomes, metacentric, submetacentric, acrocentric and telocentric chromosome are 610-10-16, 2-4-16-18, 2-4-20-14 and 6-8-10-14, respectively. Chromosome pairs 17, 19, 20 and 19 are satellite chromosomes, respectively. The X and Y chromosome are large submetacentric and small submetacentric for the masked palm civet, whereas binturong and Asian palm civet have large metacentric and small metacentric. The small-toothed palm civet contains large metacentric and small acrocentric chromosome.

As an endangered species, basic data resulting from cytogenetic studies can help to identify innate features which may lead towards the extinction of civets. Therefore, this study aims to test the normality or abnormality of chromosomal features in large-spotted civet, large Indian civet and small Indian civet.

\section{Materials and methods}

Blood samples of 2 male and 2 female large-spotted civets, 3 female large Indian civets, and 2 male and 2 female small Indian civets from Khao Khiew Zoo, Chonburi province and Dusit Zoo, Bangkok, Thailand were collected and subjected to cytogenetic studies by lymphocyte culture of whole blood samples. The cultured cells were examined with a colchicine-hypotonic-fixation-airdrying technique followed by conventional staining and G-banding with Giemsa's. Chromosomal checks using light microscopy were performed with 20 cells of each individual. 

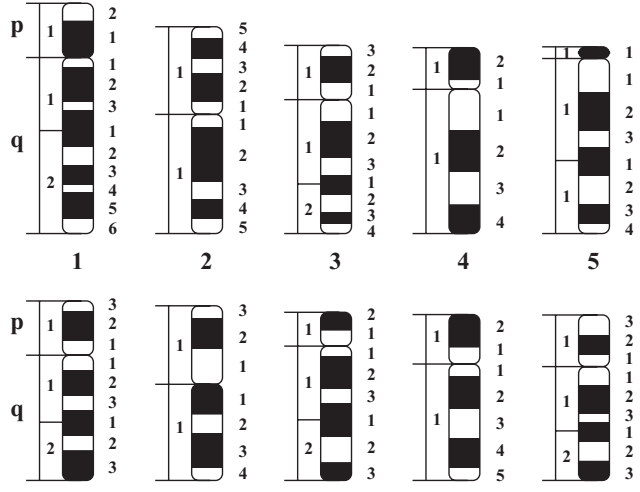

8

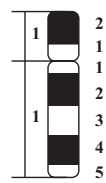

9
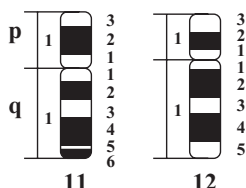

12

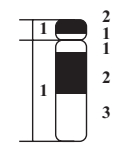

13

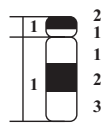

14

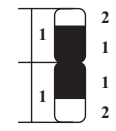

15

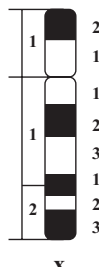

17

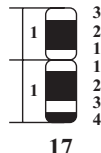

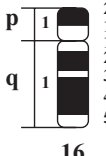

Fig. 5. Idiograms showing lengths and shapes of chromosomes for small Indian civet (Viverra zibetha) $2 n=36$.
Results

Karyotypes of these civets including large-spotted civet, large Indian civet and small Indian civet show that chromosome numbers of them are $2 n=38,38$, and 36 , respectively (Figs. 1, 2). The NF are 72 for female and 71 for male, 72 for female, and 70 for female and 69 for male. The 38 chromosomes of largespotted civet consists of 10 metacentrics, 10 submetacentrics, 12 acrocentrics and 4 telocentrics; the sex chromosomes are large submetacentric and smallest telocentric; the 15th chromosome pair is a satellite chromosome (Fig. 3). The 38 chromosomes of large Indian civet is comprised of 10 metacentrics, 10 submetacentrics, 12 telocentrics and 4 acrocentrics; the sex chromosomes are not known because of absence male sample in Thailand; the 17 th chromosome pair is a satellite chromosome (Fig. 4). The small Indian civet has 36 chromosomes, consisting of 10 metacentrics, 12 submetacentrics, 10 telocentrics and 2 acrocentrics; the sex chromosomes are large submetacentric and medium telocentric; the 17 th chromosome pair is a satellite chromosome (Fig. 5).

\section{Discussion}

Chromosome number of the small Indian civet, $2 n=36$, agrees with the previous study by Wurster and Benirschke (1967) and Wurster and Gray (1968). There is not any previous study on cytogenetics of the large-spotted civet and large Indian civet.

The NF of the small Indian civet is 70 for female and 69 for male which differs from Wurster and Benirschke (1967) and Wurster and Gray (1968). They reported that the NF was 64 for both male and female.

Idiograms of these 3 species studied indicate that chromosome sizes are gradually lowered from large to small sizes (Figs. 3, 4, 5).

The autosomes of small Indian civet, which is 10-12-10-2, different from formerly studied of Wurster and Benirschke (1967) which indicated that the autosomes were $2 n=36,26$ metacentrics and submetacentrics, and 8 acrocentrics and telocentrics. Members of family Viverridae were reported to have 22-46 metacentrics and submetacentrics, 2-20 acrocentrics and telocentrics (Wurster and Benirschke, 1967 Wurster and Gray, 1968). These indicate to have autosome number and type diversities.

The $\mathrm{X}$ chromosome of the animals belonging to subfamily Viverinae in Thailand is the large submetacentric and the $\mathrm{Y}$ chromosome is medium telocentric or smallest telocentric which differ from results found by Wurster and Benirschke (1967) and Wurster and Gray (1968). They showed that the $\mathrm{X}$ and $\mathrm{Y}$ chromosomes of small Indian civet are large metacentric and medium acrocentric.

All 3 species have 2 satellite chromosomes as genetic marker namely chromosome pair 15, 17, 
and 17.

The colors bands resulting from the G-banding staining show 143, 148 and 145 bands in different locations for each species. These variations lead to the summary that these 3 sampled species have far distance of evolutionary relationships.

\section{References}

Masashi, H. and Harumi, T. 1993. Karyotype study of the masked palm civet, Paguma lavata in Japan (Viverridae). Journal of the Mammal. Soc. Japan. 18: 39-42.

Tanomtong, A., Chaveerach, A., Phanjun, G., Kaensa, W. and Khunsook, K. 2005a. New records of chromosomal features in Indian muntjacs (Muntiacus muntjak) and Fea's muntjacs (M. feae) of Thailand. Cytologia. 70: 71-77.

- - - Sriphoom, A. and Bunjongrat, R. 2005b. Cytogenetic study on wild animal species of the subfamily Paradoxurinae in Thailand. Cytologia. 70: 249-255.

Wang, Z., Quan, G., Yie, Z. and Wag, S. 1984. Karyotype of three species of Carnivora. Acta Zool. Sinica. 30: $188-195$.

Wurster, D. H. and Benirschke, K. 1967. Chromosome number in thirty species of the Carnivora, Mammal. Chromosoma Newsletter. 8: 195-216.

— and Gray, C. W. 1968. Comparative cytogenetic studies in the order Carnivora. Chomosoma. 24: 336-382. 